Case Report

\title{
Trazodone Addition to Paroxetine and Mirtazapine in a Patient with Treatment-Resistant Depression: The Pros and Cons of Combining Three Antidepressants
}

\author{
Rui Lopes, ${ }^{1,2,3}$ José Carlos Alves, ${ }^{1}$ and Raquel Garcia Rego ${ }^{1}$ \\ ${ }^{1}$ Department of Psychiatry, Hospital do Divino Espírito Santo de Ponta Delgada, Avenida D. Manuel I, \\ Ponta Delgada, 9500-370 Azores, Portugal \\ ${ }^{2}$ Faculty of Medicine, University of Porto, Alameda Prof. Hernâni Monteiro, 4200-319 Porto, Portugal \\ ${ }^{3}$ Ciclo Básico de Medicina, Department of Biology, University of the Azores, Rua da Mãe de Deus, 9501-801 Ponta Delgada, Portugal
}

Correspondence should be addressed to Rui Lopes; rui.lopess@gmail.com

Received 7 July 2016; Revised 18 September 2016; Accepted 19 September 2016

Academic Editor: Bernardo Carpiniello

Copyright (c) 2016 Rui Lopes et al. This is an open access article distributed under the Creative Commons Attribution License, which permits unrestricted use, distribution, and reproduction in any medium, provided the original work is properly cited.

Dual antidepressant combination for treatment-resistant depression is a strategy well supported by literature and accepted in clinical practice. Rather, the usefulness of the combination of more than two antidepressants is controversial. This may be related to the possibility of higher side-effect burden and to doubts about its pharmacological effectiveness and therapeutic advantage compared to other standard treatment options. We report a relapse of moderate-to-severe depressive symptoms with insomnia that successfully remitted after the addition of trazodone to a dual combination of paroxetine and mirtazapine (in standard effective doses) in a patient with treatment-resistant depression. We also review the literature and discuss the utility of triple antidepressant combination in treatment-resistant depression. This clinical case highlights the utility of combining trazodone as a third antidepressant for the relapse of depressive symptoms after the failure of a dual antidepressant combination. Trazodone may be advantageous in patients presenting recurrence of moderate-to-severe depressive symptoms that include sleep problems and/or insomnia and may be particularly useful when benzodiazepines are not recommended. Although its use may be controversial and associated with higher risk of side-effects, more investigation is needed to determine the efficacy and safety for triple antidepressant combinations as reliable strategies for treatment-resistant depression in clinical practice.

\section{Introduction}

Major depressive disorder is associated with a high clinical, morbidity, and disability burden [1]. The number of previous episodes and subclinical residual symptoms have been identified as main predictors of recurrence [2]. Recurrent episodes in turn have been hypothetically implicated in neurodegeneration and also with cognitive dysfunction [3]. An appropriate treatment for major depressive disorder and reduction of its burden are therefore important actual key therapeutic issues. However, treatment-resistant depression or treatmentrefractory depression may assume in clinical practice a true challenge and may also have a broad definition: it can usually be presented as a failure to respond to one antidepressant or two trials with antidepressants from different pharmacological classes in adequate courses (in maximal dose for at least 6 weeks of duration), to two antidepressants in combination, or to electroconvulsive therapy (ECT) or can also be presented when there is intolerance to treatment or there is a relapse after initial response to treatment [4-6].

Current clinical and consensus guidelines recommend, in a stepwise manner, switching of the initial antidepressant after the maximal dose has failed, augmentation (adding thyroid hormone, low doses of atypical antipsychotics, or mood stabilizers), and the combination of antidepressants (antidepressant polypharmacy) [7-12]. Regarding this latter strategy, although combining two antidepressants with complementary pharmacological actions is well accepted, the triple 
antidepressant combination is, on the contrary, less described and more controversial [13-16]. Here, we present a relapse of moderate-to-severe depressive symptoms in a patient with treatment-resistant depression that was efficaciously treated after adding trazodone to a dual antidepressant combination consisting of paroxetine and mirtazapine (in standard doses); we also discuss the pros and cons of combining three antidepressants as a strategy in the management of treatmentresistant depression.

\section{Case Description}

A 42-year-old female patient was sent to our outpatient department by her family physician due to recurrent major depressive disorder symptoms that were not responding to treatment with paroxetine $20 \mathrm{mg}$ /day for 2 months. Two years before, she suffered a major depressive disorder episode, moderate-to-severe, comorbid with panic disorder that was successfully treated with paroxetine $20 \mathrm{mg}$ /day during a period of 9 months. Patient achieved full remission after 2 months and completed further 6 months of treatment. After gradually tapering paroxetine during one month, she remained euthymic in the following 12 months. More recently, the patient presented a recurrence of depressive symptoms with 3 months of evolution characterized by sadness, anxiety, anhedonia, apathy, insomnia, difficulties in carrying out work activities due to low attention and concentration, decreased sexual libido and appetite, fatigue and asthenia, and feelings of hopeless and helplessness. Patient has resumed treatment with paroxetine $20 \mathrm{mg}$ /day in the previous 8 weeks by her family physician. Due to the absence of treatment response, the patient was oriented to our outpatient department.

In the first appointment, she came with her husband and said that she has been married for 20 years and has two children who were 17 and 11 years old. She has been working as an employee of a clothing factory for the last 10 years. Her husband expressed concern and worries about her depressive state and said that at home she was "always complaining about everything." They did not associate her clinical condition with the existence of recent remarkable life events such as personal, familial, or labour problems. Nevertheless, the patient was missing days of work at her employment.

We have performed a thorough clinical evaluation. On mental state examination, the patient was oriented in time, place, and person. She presented depressed mood without suicidal ideation. Psychomotor retardation was present. No hallucinatory activity and formal or content thought disorder was detected. Insight for her morbid condition was preserved. Physical and neurological examinations were unremarkable. Routine blood assessments including full blood count (FBC), plasma glucose, and urea and electrolytes (U\&E), liver function tests (LFTs), thyroid function tests (TFTs), basic urine investigation, illicit drug screening, electroencephalogram, and a cerebral computed tomography were also performed and the results were within normal limits. There were no remarkable medical or family antecedents and no personal history of substance misuse. Also, there was no previous or current personal history of manic or hypomanic symptoms nor psychiatric disease in family history.

A major depressive disorder, recurrent episode, moderate-to-severe, was diagnosed [17]. Patient was instructed to raise paroxetine to $40 \mathrm{mg} /$ day $(20 \mathrm{mg} \mathrm{bd}$ ) and to combine mirtazapine $15 \mathrm{mg}$ at bedtime. She attended regular monthly or bimonthly scheduled meetings for monitoring and to also to perform a brief cognitive-behaviour psychotherapy. After a month, although there was a slight improvement on her clinical status, the patient maintained a moderate depressive mood with intermediate and final insomnia. Consequently, mirtazapine was raised to $30 \mathrm{mg}$ and after $4-6$ weeks of this treatment optimization her clinical remission was verified and patient returned to work.

However, after three months of paroxetine $40 \mathrm{mg} /$ day and mirtazapine $30 \mathrm{mg}$ /day dual combination (after 6 weeks of remission of depressive symptomatology), a moderateto-severe relapse of depressive symptoms, accompanied by insomnia, was verified again. Relapse is usually, by definition, the return of depressive symptoms before full remission or within the first months after remission was achieved. According to DSM-5, an interval of at least two consecutive months of full remission between a previous and a new episode must be present to be considered a recurrence [17]. In this particular case, there was a return of depressive symptoms before full remission achieved two months of length and, therefore, a relapse of the previous depressive episode must be considered.

Patient and her family were informed of the possible alternatives for managing the relapse of symptomatology including the further rising of paroxetine or mirtazapine dosage; augmentation strategies (including thyroid hormones and mood stabilizers or antipsychotics in lower doses); the addition of a third antidepressant that has sedative properties and relatively well tolerable side-effect profile, such as trazodone; or alternatively ECT. Due to the possible requirement of a concomitant hypnotic benzodiazepine or sleep-inducer to first alternatives, the patient chose with her husband the addition of trazodone for the management of moderate-tosevere relapse of depression symptomatology accompanied by insomnia. This option was also preferred in detriment of ECT. Accordingly, the patient was instructed to increase slowly the trazodone dosage $(50 \mathrm{mg}$ every three days at bed time) till reaching $150 \mathrm{mg} / \mathrm{day}$. She was also told to be aware of the possible warning side-effects and to stop trazodone if serious collateral symptoms were noticed. Instead, trazodone addition was well tolerated and a remarkable improvement in sleep symptoms was verified after reaching the dosage of $150 \mathrm{mg} /$ day. Remission of the remaining depressive symptoms was also achieved after 6 weeks and the patient recovered her self-esteem. Regular monitoring including the assessment of weight and blood pressure and electrocardiogram as well as repeated basic blood (plasma glucose, FBC, U\&E, and LFTs) and urine evaluations was performed prior to trazodone initiation and following trazodone's uptitration. Values were within normal limits and no side-effects were also detectable or reported. After 6 months, trazodone was gradually reduced (50 mg per month) and interrupted. The patient remained asymptomatic and euthymic performing paroxetine and 
mirtazapine in maintenance dosages for another 12 months and did not require any further medication adjustments.

\section{Discussion}

This case illustrates a relapse of depressive symptomatology accompanied by insomnia in a patient with treatmentresistant depression already performing a dual combination of paroxetine and mirtazapine (in standard doses) that was successfully treated by adding trazodone (as a third antidepressant). In parallel with optimization or augmentation, antidepressant combination represents one of the strategies recommended in treatment-resistant depression [18]. However, there remains important gaps concerning their costeffectiveness, longer-term benefits and also to the clinical strategies to be used when these options fail $[8,19]$. Dual combination of some antidepressants has broad support in literature: selective serotonin reuptake inhibitors (SSRIs) or venlafaxine (serotonin/noradrenaline reuptake inhibitor, SNRI) + mirtazapine (noradrenergic and specific serotoninergic antidepressant, NaSSA) or mianserin (tetracyclic antidepressant, TeCA); and SSRIs plus bupropion (norepinephrine and dopamine reuptake inhibitor, NDRI) [7, 9$11,20,21]$. Other combinations, such as monoamine oxidase inhibitors (MAOI) + tricyclic antidepressants (TCA) or SSRIs + TCA, have less support but were also formerly widely used and accepted in clinical practice $[19,22]$. More controversial, the combination of more than two antidepressants is less well studied and reported $[13-15,22]$. This may be associated with the possibility of a greater side-effect burden (including serotoninergic syndrome and syndrome of inappropriate antidiuretic hormone secretion, SIADH) and with higher clinically significant medication interactions secondary to polypharmacy [14]. Further, there are also doubts concerning its pharmacological advantage in comparison to other standard strategies. Notwithstanding, some cases of treatmentresistant or recurrent depression that were successfully treated with a triple antidepressant combination have been recently reported and may draw attention to the possibility of constituting an available and feasible therapeutic option (Table 1) $[15,22]$.

Interestingly, combining antidepressants may enhance global antidepressant efficacy through complementary and additional pharmacology mechanisms. This may constitute a valid theoretical argument that also provides support for the usefulness of this strategy in clinical practice [23]. Individually, paroxetine's antidepressant properties are dependent mainly on serotonin transporter (SERT) inhibition but also on minimal norepinephrine transporter (NET) inhibition; it also has minimal anticholinergic effects due to muscarinic type 1 receptors (M1) antagonism [24]. Mirtazapine antidepressant role depends on serotonin types $2 \mathrm{~A}, 2 \mathrm{C}$, and 3 receptors $(5-\mathrm{HT} 2 \mathrm{~A} / 2 \mathrm{C} / 3)$ antagonism and alpha2 adrenergic receptors antagonism; it also exerts histaminergic type 1 receptors (H1) antagonism (sedation and weight gain), alphal adrenergic receptors antagonism (orthostatic hypotension), and M1 antagonism (anticholinergic effects) [24]. Trazodone (serotonin antagonist/reuptake inhibitor, SARI) exerts its antidepressant effects through SERT inhibition, 5-HT2A/2C antagonism, and alpha2 adrenergic receptors antagonism; it also presents alphal adrenergic receptors antagonism (orthostatic hypotension), H1 antagonism (sedation and weight gain), and minimal anticholinergic effects [25]. Moreover, trazodone's pharmacologic effects may also depend on its active metabolite, meta-chlorophenylpiperazine (mCPP), which has affinity (mainly as an agonist) at several 5-HT receptor subtypes (including 5HT2C, 5HT3, 5HT2A, 5HT1B, 5HT1A, and 5HT1D) [26, 27]. Interestingly, mCCP agonist actions on 5-HT2A and 5-HT2C receptors might oppose trazodone antagonist actions in the same receptors and hypothetically could compromise its antidepressant effect. However, it seems that in vivo mCCP plasma and brain levels are less than $10 \%$ of trazodone and therefore are probably blocked by the parent compound [26-28].

Notably, adding mirtazapine to paroxetine augments paroxetine's SERT inhibition (and minimal NET inhibition) through mirtazapine's 5HT2A/5HT2C/5HT3/alpha2 antagonism. The third addition of trazodone may enhance SERT inhibition and 5HT2A/5HT2C and alpha2 antagonism contributing to maximizing further this dual combination. Also, it may represent an advantageous strategy in detriment of raising the dosage of the first or second antidepressants in maximal or supramaximal dosages. Therefore, the triple combination of paroxetine + mirtazapine + trazodone can have globally the following individual complimentary antidepressant effects: SERT inhibition (both paroxetine and trazodone); NET inhibition (paroxetine); 5HT3 antagonism (mirtazapine); and 5HT2A/5HT2C and alpha2 antagonism (both mirtazapine and trazodone). Although trazodone may be associated with particular rare side-effects such as priapism [16] or nocturnal enuresis [29], it is generally well tolerated, reduces symptoms of depression, and is not associated with weight gain or sexual dysfunction [25]. Importantly, its combination with other antidepressants, particularly SSRIs and SNRIs, has been used to minimize side-effects and resolve some tolerability problems such as anxiety or sexual dysfunction and also for targeting residual symptoms not well treated by SERT inhibition alone $[23,25]$. Trazodone's complementary sedative effects through $\mathrm{H} 1$ antagonism are also used for improving sleep quality and insomnia which promotes a minor use of benzodiazepines [25]. This feature is useful in particular clinical situations where the use of benzodiazepines and sleep-inducers may be discouraged (such as asthma, chronic obstructive pulmonary disease, and obstructive sleep apnoea) [25].

In this particular clinical case trazodone choice was therefore influenced due to an immediate effect on sleep and consequently addressing patient's insomnia symptoms and also in the management of other depressive symptomatology. In fact, trazodone ability to improve sleep has been considered one important augment mechanism to improve the efficacy of other antidepressants [24]. The alternatives of increasing paroxetine or mirtazapine dosage could also resolve depressive symptoms but require the prescription of an additional hypnotic benzodiazepine or sleep-inducer (at least during the initial phase). Moreover, there is evidence demonstrating that the mirtazapine or trazodone sedative effects may not 
TABLE 1: Triple antidepressant combinations (drug family, active principle, and respective dosages) described in literature with good clinical outcomes (efficacy and tolerability).

\begin{tabular}{|c|c|}
\hline Triple antidepressant combination & Study \\
\hline SSRI (escitalopram 10 mg) + TCA (amitriptyline 25 mg) + NARI (reboxetine $2 \mathrm{mg}$ ) & \multirow{3}{*}{ Restifo [15] } \\
\hline TeCA (mianserin 20 mg) + TCA (nortriptyline 75 mg) + NARI (reboxetine $1 \mathrm{mg}$ ) & \\
\hline SNRI (venlafaxine $150 \mathrm{mg}$ ) + NaSSA (mirtazapine $7.5 \mathrm{mg}$ ) + NARI (reboxetine $1 \mathrm{mg}$ ) & \\
\hline MAOI (tranylcypromine $70 \mathrm{mg}$ ) + TCA (nortriptyline $100 \mathrm{mg}$ ) + SARI (trazodone $50 \mathrm{mg}$ ) & \multirow{2}{*}{ Thomas et al. [22] } \\
\hline MAOI (phenelzine $60 \mathrm{mg}$ ) + TCA (nortriptyline $150 \mathrm{mg}$ ) + SARI (trazodone $400 \mathrm{mg}$ ) & \\
\hline SSRI (paroxetine $40 \mathrm{mg}$ ) + NaSSA (mirtazapine $30 \mathrm{mg}$ ) + SARI (trazodone $150 \mathrm{mg}$ ) & Present report \\
\hline
\end{tabular}

increase with higher dosages $[30,31]$, but possibly one way to potentiate their sedative effect may be combining them. Further, mirtazapine could also be discontinued when trazodone was started, as both may have identical antidepressants effects (5HT2A/5HT2C/alpha2 antagonism) [24, 25]. Notwithstanding, mirtazapine can also have an additional antidepressant effect through 5HT3 antagonism [24], therefore contributing through this way to maximizing the triple antidepressant combination of paroxetine, mirtazapine, and trazodone.

Trazodone was increased gradually in accordance with patient tolerance accompanied with appropriate clinical monitoring and particular attention to side-effects burden. Following 6 months of successful treatment and clinical remission, trazodone was reduced and stopped gradually, as recommended by NICE guidelines which consider that the augmentation agent should be stopped at first instance [9].

Despite the presumptive theoretical advantage of combining three different antidepressants with complementary pharmacological profiles, its usage in clinical practice may rather be influenced by several factors including the clinical presentation itself, the personal clinician prescription practices, and, last but not the least, the choice of a clearly informant patient on the possible available treatment alternatives. Interestingly, psychiatrists' attitude about antidepressants prescription practices in clinical settings is a matter not entirely well studied [32]. Nevertheless, a convenient knowledge of general pharmacology should be present when decision on polypharmacy is made. Accordingly, higher risk of clinically significant medication interactions associated with antidepressant polypharmacy may result from inhibition and induction of cytochrome P450 (CYP) hepatic enzymes, which may lead to antidepressant toxicity or to a subtherapeutic effect, respectively. Particularly, paroxetine's inhibition of CYP1A2 and CYP3A enzymes may result in higher plasma levels of mirtazapine; further, paroxetine also inhibits CYP2D6 that may lead to higher trazodone plasma levels. Together, these additional effects may undergo reciprocal increased antidepressant levels and subsequent toxicity such as higher risk of serotonin syndrome, although this may be rare in clinical practice [10]. Nevertheless, it has also been documented that serotonin syndrome risk might increase almost exponentially with the use of antidepressants acting with different mechanisms on the same pathway and therefore this risk should be not negligible [33].

The patient, however, did not present any signs or symptoms of serotonin syndrome and also pharmacologic interactions were presumably not verified on this clinical case due to the absence of side-effects and reported toxicity. In general, although these interactions can be pharmacologically wellpredicted in clinical practice, it has been recently documented that important genetic polymorphisms associated with cytochromes enzymes may lead to variable pharmacokinetic profiles (ultrarapid or poor metabolisers) [10]. Moreover, intraindividual variability on antidepressant treatment outcomes may also be dependent on different pharmacodynamic responses (such as high functioning serotonin transporter genotypes) [34]. A recent meta-analysis has demonstrated that combining antidepressants was statistically significantly superior compared to monotherapy [35]. However, the percentage of drop-outs related to adverse events with antidepressant combination was also higher [35], which therefore may raise concerns about treatment compliance. Due to higher probability of pharmacokinetic and pharmacodynamic interactions when antidepressant polypharmacy is prescribed, more careful monitoring should be therefore a practical concern to prevent unexpected or rare side-effects. This is especially important in certain patients groups such as those with renal, hepatic, and cardiac impairments, those with epilepsy, pregnant or breast-feeding women, the elderly, and children.

Moreover, pharmacokinetic and/or pharmacodynamic tolerance effects resulting from chronic exposure to antidepressants have also been hypothesized to be related to the development of antidepressant tachyphylaxis or tolerance (loss of antidepressant efficacy when in maintenance treatment with the same drug and dosage regimen) [36, 37]. Noncompliance or nonadherence to drug treatment has also been associated with depressive symptomatology relapse [36, 37] and although this was not evaluated by patient plasma analysis, it was closely monitored by her husband and confirmed in each visit. Although these two factors could possibly be associated with the initial depressive relapse, in our patient this was probably more associated with the worsening of the underling depressive episode $[36,37]$. 


\section{Conclusions}

This case report illustrates a relapse of depressive symptoms with insomnia that was successfully remitted after the addition of trazodone in a patient with treatment-resistant depression already performing a previous dual antidepressant combination of mirtazapine and paroxetine in standard antidepressant doses. The combination of trazodone as a third antidepressant in this case report not only allowed a rapid control of insomnia but also was efficacious for the management of other depressive symptoms and was well tolerated.

Trazodone addition as a triple antidepressant combination strategy may be used in treatment-resistant or recurrent depression patients in whom standard treatment options including dual antidepressant combination did not produce a successful response. Accordingly, combining trazodone with other antidepressants with complementary pharmacological actions can globally enhance antidepressant effect. This may be achieved by targeting residual symptoms not well treated by a single antidepressant effect such as SERT inhibition performed by SSRIs or through exerting other additional pharmacological actions. Interestingly, it may also resolve some tolerability issues by reducing SSRI or SNRI associated sideeffects such as sexual dysfunction.

Moreover, combining trazodone with other antidepressants with complementary pharmacological actions might represent a more sustainable and tolerable option to the alternative of individually increasing the dose of the previous dual antidepressant combination or even other strategies including antidepressant augmentation (thyroid hormones, low doses of mood stabilizers, or antipsychotics) or ECT. In addition, it may also be a particularly useful treatment option in selected treatment-resistant depression cases presenting with insomnia in which the prescription of benzodiazepines and other sleep-inducers is not recommended.

However, some important pharmacologic issues should be considered when triple antidepressant combination is prescribed. Accordingly, antidepressant polypharmacy warrants a close clinical supervision and monitoring due to higher risk of pharmacokinetic and pharmacodynamic drug interactions of which it is essential to be aware. These may be associated with higher secondary side-effects and toxicity including the risk of serotonin syndrome. Prior to the addition and administration of a third antidepressant, an analytical checkup is therefore essential (including plasma glucose, FBC, $\mathrm{U} \& \mathrm{E}$, and LFTs); thereafter, close, regular, and careful clinical monitoring including basic blood and biochemical reassessments during third antidepressant's titration should also be performed to prevent side-effects.

Other major limitations to this case report are intrinsically related to the naturalistic based observational study which does not allow the control and determination of the possible variables that may interfere with the treatment outcome. Further, patient adherence to treatment was not evaluated in plasma analysis and severity of depressive symptoms as well clinical response to treatment was not assessed with the aid of any clinical scale.

More investigation is needed to clearly define the place of triple antidepressant combination as a strategy in the management of treatment-resistant depression. Therefore, large, prospective, and well-controlled trials are needed for a rigorous establishment of risk-benefit ratio, as well as efficacy, tolerability, and safety issues and also for comparing directly with other existing alternatives.

\section{Competing Interests}

The authors report no conflict of interests.

\section{Acknowledgments}

The authors thank Dulce Raposo from the Hospital do Divino Espírito Santo Facilities and Equipment Service, for her assistance with the English revision.

\section{References}

[1] Global Burden of Disease Study 2013 Collaborators, "Global, regional, and national incidence, prevalence, and years lived with disability for 301 acute and chronic diseases and injuries in 188 countries, 1990-2013: a systematic analysis for the Global Burden of Disease Study 2013," The Lancet, vol. 386, no. 9995, pp. 743-800, 2015.

[2] F. Hardeveld, J. Spijker, R. De Graaf, W. A. Nolen, and A. T. F. Beekman, "Prevalence and predictors of recurrence of major depressive disorder in the adult population," Acta Psychiatrica Scandinavica, vol. 122, no. 3, pp. 184-191, 2010.

[3] H. K. Kim, P. V. Nunes, K. C. Oliveira, L. T. Young, and B. Lafer, "Neuropathological relationship between major depression and dementia: a hypothetical model and review," Progress in NeuroPsychopharmacology and Biological Psychiatry, vol. 67, pp. 51-57, 2016.

[4] D. F. Ionescu, J. F. Rosenbaum, and J. E. Alpert, "Pharmacological approaches to the challenge of treatment-resistant depression," Dialogues in Clinical Neuroscience, vol. 17, no. 2, pp. 111-126, 2015.

[5] M. T. Berlim and G. Turecki, "What is the meaning of treatment resistant/refractory major depression (TRD)? A systematic review of current randomized trials," European Neuropsychopharmacology, vol. 17, no. 11, pp. 696-707, 2007.

[6] M. T. Berlim and G. Turecki, "Definition, assessment, and staging of treatment-resistant refractory major depression: a review of current concepts and methods," Canadian Journal of Psychiatry, vol. 52, no. 1, pp. 46-54, 2007.

[7] M. H. Trivedi, M. Fava, S. R. Wisniewski et al., "Medication augmentation after the failure of SSRIs for depression," The New England Journal of Medicine, vol. 354, no. 12, pp. 1243-1252, 2006.

[8] A. J. Rush, M. H. Trivedi, S. R. Wisniewski et al., "Acute and longer-term outcomes in depressed outpatients requiring one or several treatment steps: a STAR ${ }^{*} \mathrm{D}$ report," The American Journal of Psychiatry, vol. 163, no. 11, pp. 1905-1917, 2006.

[9] National Institute for Health and Clinical Excellence, "Depression: the treatment and management of depression in adults (update)," Clinical Guideline 90, 2009, http://www.nice.org.uk.

[10] D. Taylor, C. Paton, and S. Kapur, The Maudsley Prescribing Guidelines in Psychiatry, John Wiley \& Sons, London, UK, 12th edition, 2015.

[11] P. J. McGrath, J. W. Stewart, M. Fava et al., "Tranylcypromine versus venlafaxine plus mirtazapine following three failed 
antidepressant medication trials for depression: a STAR $^{*} \mathrm{D}$ report," The American Journal of Psychiatry, vol. 163, no. 9, pp. 1531-1541, 2006.

[12] A. A. Nierenberg, M. Fava, M. H. Trivedi et al., "A comparison of lithium and $\mathrm{T}_{3}$ augmentation following two failed medication treatments for depression: a STAR* D report," American Journal of Psychiatry, vol. 163, no. 9, pp. 1519-1530, 2006.

[13] L. M. Martín-López, J. E. Rojo, K. Gibert et al., “The strategy of combining antidepressants in the treatment of major depression: clinical experience in spanish outpatients," Depression Research and Treatment, vol. 2011, Article ID 140194, 8 pages, 2011.

[14] A. Wiggins, T. Balasubramanian, and A. Ferraro, "Hyponatraemia and confusion in a 70-year-old female when bupropion was added to dothiepin and escitalopram," Australasian Psychiatry, vol. 23, no. 5, pp. 507-509, 2015.

[15] S. Restifo, "Walking on thin ice: the use of triple antidepressant combinations in refractory melancholic depression," Australasian Psychiatry, vol. 20, no. 2, pp. 134-137, 2012.

[16] G. Jayaram and P. Rao, "Safety of trazodone as a sleep agent for inpatients," Psychosomatics, vol. 46, no. 4, pp. 367-369, 2005.

[17] American Psychiatric Association (APA), Diagnostic and Statistical Manual of Mental Disorders: DSM-5, American Psychiatric Association, Arlington, Va, USA, 5th edition, 2013.

[18] M. E. Thase, "Antidepressant combinations: cutting edge psychopharmacology or passing fad?" Current Psychiatry Reports, vol. 15, no. 10, article 403, 2013.

[19] K. R. Connolly and M. E. Thase, "If at first you don't succeed: a review of the evidence for antidepressant augmentation, combination and switching strategies," Drugs, vol. 71, no. 1, pp. 43-64, 2011.

[20] L. L. Carpenter, S. Yasmin, and L. H. Price, "A double-blind, placebo-controlled study of antidepressant augementation with mirtazapine," Biological Psychiatry, vol. 51, no. 2, pp. 183-188, 2002.

[21] M. Ferreri, F. Lavergne, I. Berlin, C. Payan, and A. J. Puech, "Benefits from mianserin augmentation of fluoxetine in patients with major depression non-responders to fluoxetine alone," Acta Psychiatrica Scandinavica, vol. 103, no. 1, pp. 66-72, 2001.

[22] S. J. Thomas, M. Shin, M. G. McInnis, and J. R. Bostwick, "Combination therapy with monoamine oxidase inhibitors and other antidepressants or stimulants: strategies for the management of treatment-resistant depression," Pharmacotherapy, vol. 35, no. 4, pp. 433-449, 2015.

[23] S. M. Stahl, C. Lee-Zimmerman, S. Cartwright, and D. A. Morrissette, "Serotonergic drugs for depression and beyond," Current Drug Targets, vol. 14, no. 5, pp. 578-585, 2013.

[24] S. M. Stahl and N. Muntner, "Antidepressants," in Stahl's Essential Psychopharmacology: Neuroscientific Basis and Practical Applications, S. M. Stahl and N. Muntner, Eds., chapter 7, pp. 284-369, Cambridge University Press, Cambridge, UK, 4th edition, 2013.

[25] A. Fagiolini, A. Comandini, M. C. Dell'Osso, and S. Kasper, "Rediscovering trazodone for the treatment of major depressive disorder," CNS Drugs, vol. 26, no. 12, pp. 1033-1049, 2012.

[26] S. M. Stahl, "Mechanism of action of trazodone: a multifunctional drug," CNS Spectrums, vol. 14, no. 10, pp. 536-546, 2009.

[27] M. Maes, H. Westenberg, E. Vandoolaeghe et al., "Effects of trazodone and fluoxetine in the treatment of major depression: therapeutic pharmacokinetic and pharmacodynamic interactions through formation of meta-chlorophenylpiperazine,"
Journal of Clinical Psychopharmacology, vol. 17, no. 5, pp. 358364, 1997.

[28] K. Otani, K. Mihara, N. Yasui et al., "Plasma concentrations of trazodone and $\mathrm{m}$-chlorophenylpiperazine at steady state can be predicted from those after an initial dose of trazodone," Progress in Neuro-Psychopharmacology and Biological Psychiatry, vol. 21, no. 1, pp. 239-244, 1997.

[29] R. Lopes, J. Matias, and R. C. de Melo, "Trazodone-induced nocturnal enuresis," Psychiatry Research, vol. 235, pp. 211-212, 2016.

[30] M. Savarese, M. Carnicelli, V. Cardinali et al., "Subjective hypnotic efficacy of trazodone and mirtazapine in patients with chronic insomnia: a retrospective, comparative study," Archives Italiennes de Biologie, vol. 153, no. 2-3, pp. 231-238, 2015.

[31] S. M. Stahl, Stahl's Essential Psychopharmacology: Prescriber's Guide, Cambridge University Press, Cambridge, UK, 5th edition, 2014.

[32] N. M. Davies, D. Gunnell, K. H. Thomas, C. Metcalfe, F. Windmeijer, and R. M. Martin, "Physicians' prescribing preferences were a potential instrument for patients' actual prescriptions of antidepressants," Journal of Clinical Epidemiology, vol. 66, no. 12, pp. 1386-1396, 2013.

[33] E. W. Boyer and M. Shannon, "The serotonin syndrome," The New England Journal of Medicine, vol. 352, no. 11, pp. 1112-1120, 2005.

[34] C. Fabbri and A. Serretti, "Pharmacogenetics of the efficacy and side effects of antidepressant drugs," in Genetic Influences on Response to Drug Treatment for Major Psychiatric Disorders, K. J. Rybakowski and A. Serretti, Eds., pp. 39-54, Springer, Cham, Switzerland, 2016.

[35] J. Henssler, T. Bschor, and C. Baethge, "Combining antidepressants in acute treatment of depression: a meta-analysis of 38 studies including 4511 patients," Canadian Journal of Psychiatry, vol. 61, no. 1, pp. 29-43, 2016.

[36] M. E. Thase, "Preventing relapse and recurrence of depression: a brief review of therapeutic options," CNS Spectrums, vol. 11, no. 12, supplement 15, pp. 12-21, 2006.

[37] S. D. Targum, "Identification and treatment of antidepressant tachyphylaxis," Innovations in Clinical Neuroscience, vol. 11, no. 3-4, pp. 24-28, 2014. 


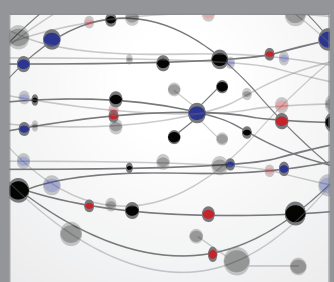

The Scientific World Journal
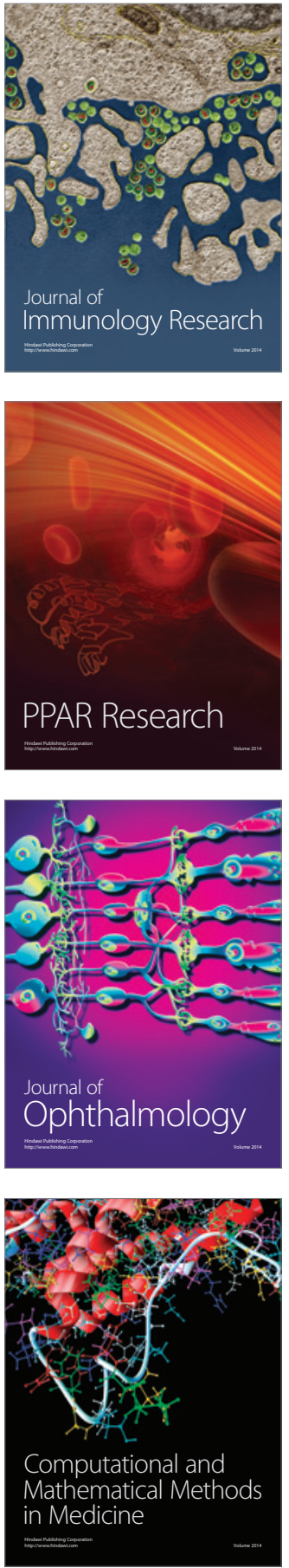

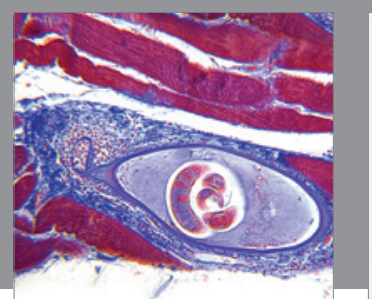

Gastroenterology Research and Practice

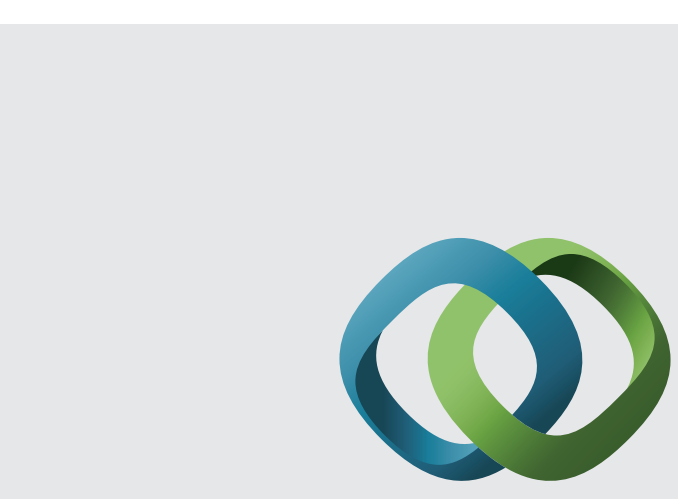

\section{Hindawi}

Submit your manuscripts at

http://www.hindawi.com
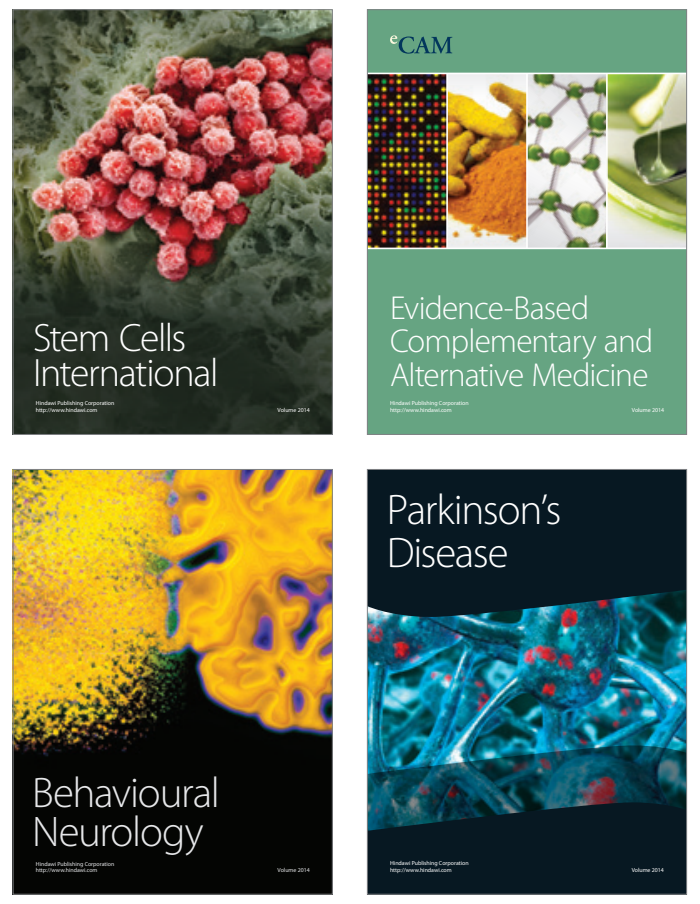
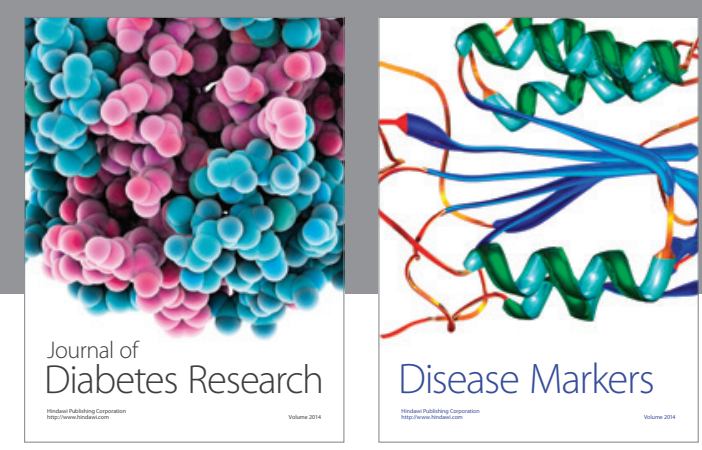

Disease Markers
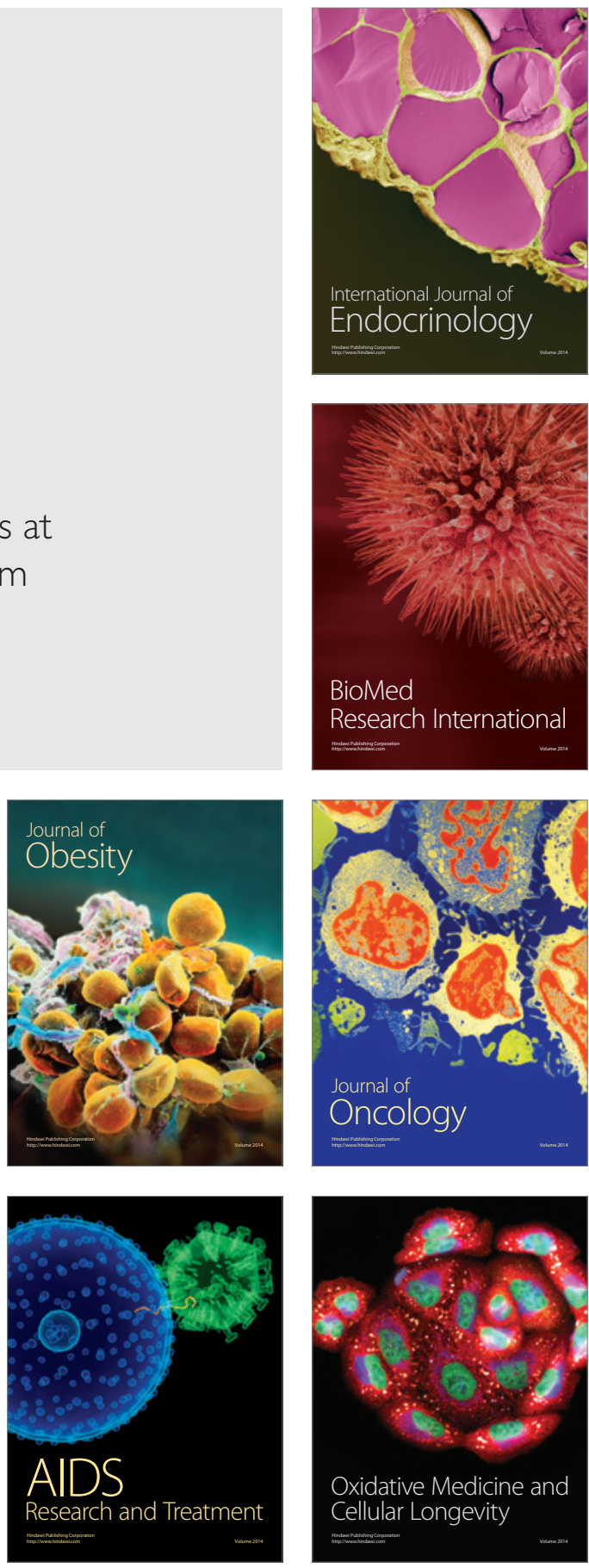